\section{UM OLHAR ALTERNATIVO SOBRE A AVALIAÇÃO DA INVESTIGAÇÃO CIENTÍFICA: A PERSPECTIVA DO IMPACTO SOCIETAL PELA ANÁLISE DAS VIAS DE IMPACTO}

\author{
AN ALTERNATIVE LOOK AT SCIENTIFIC \\ RESEARCH EVALUATION: THE PERSPECTIVE \\ OF SOCIETAL IMPACT BY AN IMPACT \\ PATHWAYS ANALYSIS
}

Fernanda Laura Medeiros Justino Lima dos Santos fernandalimast@gmail.com Doutoranda em Governação, Conhecimento e Inovação pela Univ Coimbra, Centro de Estudos Sociais, Faculdade de Economia da Universidade de Coimbra, Portugal. Servidora da Universidade Federal de Pernambuco, Brasil.

\section{RESUMO}

Este ensaio tem como objetivo apresentar uma ótica diferente sobre a avaliação da investigação científica, abordando questões relacionadas aos impactos originados pelo processo de geração e disseminação do conhecimento. Para embasar a proposta por um olhar alternativo, foi apresentada uma discussão teórica sobre a avaliação do impacto da pesquisa, seguida pela avaliação de impacto societal e a abordagem das vias de impacto. A primeira parte trata da importância em observar e seguir os elementos que baseiam os processos avaliativos, bem como o propósito pelo qual eles são estabelecidos. Na segunda etapa, entra-se na temática de orientação para o impacto, que é essencial para o argumento central por um olhar alternativo, e trata de questões que vão além da exposição de resultados, como a articulação entre atores e a existência de diferentes dimensões de impacto. Finalizando a discussão, apresenta-se a abordagem da análise das vias de impacto da pesquisa, uma perspectiva que permite uma avaliação rica e robusta da investigação e suas características. Em conclusão, defende-se que avaliar o impacto societal por meio da análise das vias de impacto é uma alternativa relevante e positiva para esfera científica. Como sugestão de estudos futuros, propõe-se que sejam explorados métodos e ferramentas que possam retratar a realidade por meio desse prisma, bem como os aspectos relacionados às diferentes áreas do conhecimento.

Palavras-chave: Avaliação da Pesquisa. Vias de Impacto. Impacto Societal. Metodologias Não Tradicionais. 


\section{ABSTRACT}

This essay aims to present a different perspective on the evaluation of scientific research, addressing impact-related issues caused by the process of knowledge generation and dissemination. To support the proposal for an alternative look, a theoretical discussion on impact evaluation was presented, followed by societal impact evaluation and the impact pathways approach. The first part deals with the importance of observing and following the elements that sustain evaluative processes as well as the purpose for which it is established. The second part addresses the theme of impact orientation, which is essential to the central argument for an alternative look and deals with issues that go beyond the exposure of results, such as the articulation between actors and the existence of different dimensions of impact. Finalizing the discussion, the approach of research impact pathways analysis is presented, a perspective that allows for a rich and robust evaluation of the research and its characteristics. In conclusion, it is argued that assessing societal impact through impact pathways analysis is a relevant and positive alternative for the scientific sphere. As a suggestion for future studies, it is proposed that methods and tools be explored that can portray reality through this prism, as well as aspects related to different areas of knowledge.

Keywords: Impact Pathways. Non-traditional Methodologies. Societal Impact. Research Evaluation.

\section{INTRODUÇÃO}

Tem-se estabelecido, em nível global, um aumento da cobrança pela comprovação de retornos gerados pelos investimentos em pesquisa, especialmente por meio de recursos públicos. Não se pode concentrar o propósito das avaliações científicas em comprovação de publicações, sob a premissa de que a geração de conhecimento em si é um propósito isolado. Argumenta-se, nesse ensaio, que isso não é suficiente e merece uma reflexão mais aprofundada.

Entretanto, o contexto atual tem demonstrado um conjunto de esforços para que a produção científica seja direcionada para uma maior aplicabilidade das pesquisas, sem perder a orientação para a qualidade. Esse movimento leva a uma constante pressão sobre o desempenho da investigação e a capacidade de comprovar seus benefícios diretos (GUNN; MINTROM, 2017).

Nesse sentido, é possível perceber um corpo de literatura que explora abordagens, metodologias e técnicas de avaliação das pesquisas, que busca contribuir com o desempenho da investigação, fazendo que os pesquisadores e gestores tenham instrumentos direcionados aos impactos que almejam. Os principais temas abordados são a alocação de recursos, a excelência na investigação e a reputação das instituições (THOMAS et al., 2020).

A perspectiva do impacto societal proporciona a visão de todos os elementos que a investigação pode contribuir com a sociedade por meio de diferentes dimensões e pela associação de múltiplos resultados e relações. Entre os aspectos considerados, estão as questões de cunho econômico, a construção de melhores ambientes sociais, a redução de desigualdades, a diminuição do desgaste no planeta e as condições de saúde pública. Esses são exemplos que demonstram uma nova agenda para vários atores na sociedade (JOLY; MATT, 2017; TERÄMÄ et al., 2016).

Um formato de avaliação que estabeleça parâmetros diferentes dos tradicionais, explorando os impactos que vão além da área científica, pode complementar a abordagem dominante da avaliação científica. Enquanto uma avaliação tradicional se centra no impacto das publicações e das revistas correspondentes, que já possuem algoritmos e processos estabelecidos, para realizar uma avaliação complexa de impacto societal, é necessário que haja uma combinação entre metodologia apropriada, acompanhamento sistemático e tempo para a devida condução do processo. 
As demandas das agências públicas de financiamento têm uma forte influência nessa temática, uma vez que recebem pressões sociais e políticas para comprovar a boa gestão dos recursos alocados, os quais são direcionados para o fomento de pesquisas relevantes e contributivas. Os posicionamentos dos atores e os valores envolvidos no processo de avaliação afetam a forma como os trabalhos são apresentados, quais são selecionados, os indicadores que são acompanhados ao longo do tempo, bem como a cultura de avaliação dentro da comunidade acadêmica.

Para se fomentar uma cultura de orientação ao impacto nas pesquisas, espera-se que a abordagem de avaliação seja capaz de incorporar o processo de pesquisa e de produção e disseminação do conhecimento como um todo, considerando sua complexidade e englobando estágios intermediários, de forma que se produzam informações relevantes para a gestão de projetos e programas futuros (HILL, 2016; SPRINGER-HEINZE et al., 2003).

Por essa razão, defende-se a necessidade de um olhar alternativo sobre a avaliação da investigação científica, por meio de uma análise diferente da tradicional. A perspectiva do impacto societal atende à demanda por avaliação, enquanto, ao mesmo tempo, proporciona um entendimento do propósito da produção de conhecimento para a sociedade como um todo.

Este trabalho foi desenvolvido no formato de um ensaio teórico, cujo propósito foi o de defender a tese sobre a avaliação do impacto societal por meio da abordagem das vias de impacto como uma alternativa para identificação dos impactos gerados pelas pesquisas científicas. Para atender a esse objetivo, foi conduzida uma extensa pesquisa bibliográfica sobre o tema abordado. Foram utilizadas três plataformas de busca para identificar artigos publicados na área, que foram a Scopus, o Google Acadêmico e a B-On. Não foi conduzida uma revisão sistemática, os artigos foram selecionados para leitura por meio do conteúdo dos resumos. Em alguns casos, foi utilizada a técnica do snowball para selecionar outras bi- bliografias que tratavam diretamente da análise de impacto societal.

\section{AVALIAÇÃO DE IMPACTO DA PESQUISA}

Como outros aspectos da vida social, a pesquisa científica passou a incorporar a tecnicidade por influência do pensamento econômico, conforme Ball (2015). As demandas apontadas pelo sistema de financiamento e de contabilidade pública interferem em todo o sistema de investigação, seja por meio das avaliações de desempenho, dos rankings, dos incentivos variáveis de remuneração, parcerias público-privadas, seja por outros mecanismos.

O objetivo de uma avaliação de impacto não deve ser apenas o de adicionar eficiência à distribuição de recursos com métricas simplificadas, mas também os questionamentos sobre como e por que determinados resultados e impactos são alcançados. É necessário que haja uma compreensão mais profunda dos processos relacionados ao impacto, e uma vez que sejam desenvolvidas capacidades para conduzir as avaliações, que seus resultados passem a ser utilizados para aprimorar os projetos e programas futuros, em nível de gestão, complexidade e desempenho (SPRINGER-HEINZE et al., 2003; TERRAPON-PFAFF et al., 2018).

Fazendo uma síntese das razões que podem levar à condução de uma avaliação de impacto, Molas-Gallart (2012) argumenta que elas podem ser agrupadas em três propósitos centrais, que são a distribuição e alocação de recursos, o desenvolvimento de melhores práticas para as atividades de pesquisa e o controle do uso de recursos públicos.

Com base no objetivo da avaliação, muitas decisões são tomadas, inclusive, sobre os métodos e as técnicas que serão utilizados. É possível que se estabeleça uma visão limitada do papel da pesquisa e dos impactos a ela relacionados, especialmente com a escolha de indicadores de análise que permitam simplificar comparações ou identificar alguma caraterística em particular (MOLAS-GALLART, 2015). 
Embora a avaliação das pesquisas seja um elemento fundamental para a ação governamental de, praticamente, todos os países, uma questão importante se trata de como conduzir essas avaliações sem influenciar, negativamente, a excelência das pesquisas e a autonomia científica. Além disso, o escopo da avaliação agora vai além dos resultados acadêmicos tradicionais, como as publicações e patentes, incluindo os impactos fora desse ambiente, buscando compreender como os elementos da investigação têm relação causal com aspectos econômicos, políticos, sociais, ambientais etc. (GUNN; MINTROM, 2017).

Os impactos gerados pela investigação, especialmente aquela fomentada com recursos públicos, podem ter efeitos diretos ou indiretos e abrangem diferentes arenas, como as da economia, da política, do meio ambiente e da saúde. Matt et al. (2017) lembram que esses impactos se relacionam com os vários componentes da investigação, desde a produção do conhecimento até a infraestrutura física.

A identificação desses elementos indica a necessidade de um olhar mais cuidadoso para o processo de avaliação, buscando não somente um mecanismo abrangente que permita a comprovação do bom uso dos recursos investidos, mas que tenha a capacidade de capturar, da melhor forma possível, o valor e os benefícios gerados pela investigação científica para a sociedade.

Os modelos de avaliação baseados em teoria procuram desenvolver suposições baseadas no arcabouço teórico e examinar as evidências empíricas, confrontando categorias e hipóteses dentro da lógica dos atores envolvidos no processo de desenvolvimento das pesquisas sobre os resultados e os potenciais impactos alcançados. O objetivo de desenvolver esses modelos é capturar informações e perspectivas que vão além de uma análise setorizada (SPRINGER-HEINZE et al., 2003).

A discussão sobre os impactos da pesquisa pode-se tornar bastante ampla, uma vez que existem vários aspectos para explorar e ter em conta; alguns deles foram apontados por Terämä et al. (2016): as relações entre os ato- res, estejam eles dentro ou fora da esfera acadêmica; a especificidade do trabalho, que varia de acordo com a fronteira entre teoria e prática; a busca por um equilíbrio entre o custo e o retorno dos projetos e programas; a orientação para o impacto e sua influência sobre o caráter do conhecimento que é produzido; e mesmo os ris$\cos \mathrm{e}$ ameaças à autonomia dos pesquisadores.

Os pontos de encontros, a dinâmica entre as etapas e os atores envolvidos nesse processo ainda são pouco conhecidos, lembram Gunn e Mintrom (2017). É preciso desenvolver análises que explorem esse contexto de uma forma que seja abrangente, mas sem ser genérica, de modo a proporcionar uma reflexão mais profunda sobre impacto. Essas questões serão abordadas em um estudo futuro, com planejamento já em execução.

Pelas razões aqui apresentadas, novos formatos de avaliação de pesquisa têm sido exigidos, com o intuito de englobar essas mudanças conceituais na produção e disseminação de conhecimento. Para enfrentar problemas sociais sistêmicos, os quais muitas pesquisas buscam soluções, demanda-se uma compreensão das múltiplas causas e consequências e das características inerentes de incerteza e complexidade de um longo processo de mudança (MATT et al., 2017).

\section{AVALIAÇÃO DE IMPACTO SO- CIETAL}

No planejamento de uma pesquisa, é preciso direcionar, intencionalmente, a estratégia para atividades e abordagens que possam proporcionar um maior impacto dos resultados a serem produzidos. Esse é um entendimento que vem sendo considerado há algum tempo no meio acadêmico, como pode ser visto em Briones et al. (2004). Com a busca por mais destaque sobre os impactos societais, esses aspectos passaram a estar, cada vez mais, presentes tanto nas propostas de investigação, como nas chamadas de financiamento.

$\mathrm{O}$ uso da nomenclatura societal quando se refere ao impacto da pesquisa é de forma 
que possa haver uma compreensão ampla, que não se limita à aplicabilidade de um produto ou serviço resultante da investigação. Ademais, busca-se identificar um impacto global direcionado à sociedade, que vai além da categorização tradicional determinada pelos formatos tradicionais de avaliação.

O impacto societal pode ser identificado por meio de outras terminologias, que, embora tratadas como equivalentes, não abordam o conceito na totalidade. As principais delas são valor público, transferência de conhecimento, aplicabilidade e relevância social. Essas expressões, segundo Bornmann (2013), referem-se à avaliação dos retornos social, cultural, ambiental e econômico dos resultados ou produtos de investigações financiadas com recursos públicos. Fora isso, não se pode deixar de considerar os aspectos políticos e as relações com os atores governamentais.

Dessa forma, neste ensaio, apresenta-se a compreensão do impacto societal como uma mudança causada pela intervenção de um novo conhecimento que atinge alguma ou várias esferas da sociedade, direta ou indiretamente, nas dimensões econômica, social, política, ambiental, cultural ou organizacional (HILL, 2016; JOLY et al., 2015; MATT et al., 2017; TERRAPON-PFAFF et al., 2018).

A amplitude proposta por esse tipo de avaliação leva a uma reflexão sobre a possibilidade de identificar um padrão temporal. A proposição de um modelo geral que determine o tempo ideal para avaliar esses impactos das pesquisas não parece razoável, uma vez que as características do projeto influenciam bastante em como e quando a sociedade recebe os benefícios gerados pelos resultados de uma investigação, os quais são absorvidos de forma heterogênea pelos usuários e atores envolvidos. Enquanto alguns impactos podem ser quase imediatos, especialmente quando há tecnologia específica envolvida, outros podem levar anos para se estabelecer (GUNN; MINTROM, 2017; HILL, 2016).

A avaliação dos impactos societais ganhou uma nova dimensão quando passou a in- corporar os sistemas nacionais de avaliação de pesquisa. Uma das experiências mais expressivas é a do Reino Unido, por meio do Research Excellence Framework (REF), que teve sua edição mais recente em 2014 e deverá finalizar a próxima ainda em 2021. Esse processo é uma das maiores referências de avaliação sistemática, também sendo possível identificar iniciativas simulares na Austrália e nos Países Baixos (BARRATT; BARRATT; BRENTON, 2016; GRANT et al., 2010).

Embora haja uma amplitude de questionamentos que podem ser abordados, o objetivo aqui é demonstrar que existe um olhar alternativo para guiar e conduzir avaliações de pesquisas científicas com base no impacto societal da investigação. Na busca por soluções e respostas, foram utilizadas as teorias relacionadas à abordagem das vias de impacto, que têm uma enorme capacidade de proporcionar mais entendimento sobre como as pesquisas acontecem, os atores envolvidos, os acontecimentos-chave etc.

\section{ABORDAGEM DAS VIAS DE IM- PACTO}

Considerando a necessidade de explorar diversos elementos sobre os processos de geração e disseminação do conhecimento e da avaliação de impactos de pesquisa, foi delineado o modelo de Análise das Vias de Impacto (Impact Pathways). Este tem o intuito de proporcionar a identificação das diferentes etapas da criação de impacto, dos atores e recursos envolvidos e a mudança longa e complexa do conhecimento para resultados e impactos da pesquisa (DOUTHWAITE et al., 2003; JOLY et al., 2015; MATT et al., 2017; WALKER et al., 2008).

Originalmente, o modelo foi proposto na Agência Alemã de Desenvolvimento (GTZ), conforme Kuby (1999), e, posteriormente, refinado para utilização na avaliação de impacto das pesquisas de agricultura Consultative Group on International Agricultural Research (CGIAR), como visto no trabalho de Douthwa- 
ite et al. (2003). Atualmente, existem algumas abordagens desenvolvidas com base na lógica das vias de impacto de pesquisa, como o Socio-economic Analysis of the Impacts of Public Agricultural Research (ASIRPA), apresentado, detalhadamente, por Joly et al. (2015) e o Public Value Mapping (PVM), conforme Bozeman e Sarewitz (2011).

A análise das vias de impacto tem o objetivo de identificar ligações causais entre uma investigação e seus benefícios esperados e alcançados. $\mathrm{O}$ modelo permite que os pesquisadores formulem hipóteses sobre os caminhos pelos quais as atividades relacionadas à investigação devem proporcionar impacto, além de identificar quais mudanças e relações se desenvolvem ao longo do processo. Por meio desse constructo, também é possível determinar os indicadores mais apropriados para medir os diferentes estágios e resultados existentes (BRIONES et al., 2004; MATT et al., 2017; SPRINGER-HEINZE et al., 2003).

A metodologia de avaliação das vias do impacto da investigação (Impact Pathway Evaluation) foi, originalmente, proposta para acontecer em duas etapas. A primeira delas ocorre enquanto o projeto está sendo conduzido, a título de planejamento, monitoramento e avaliação do que foi planejado pelos próprios membros da equipe. A segunda acontece após um tempo da conclusão da pesquisa, sendo uma avaliação de impacto ex post, guiada por uma entidade independente, não envolvida com a condução dos trabalhos (DOUTHWAITE et al., 2003).

Esta última etapa, conforme Walker et al. (2008), subdivide-se em dois momentos, para captar impactos intermediários, mais associados com tecnologia, e impactos mais abrangentes, maioritariamente de cunho socioambiental. Essa separação permite identificar se houve uma amplificação dos impactos de determinado projeto após um período mais significativo, ou seja, alguns anos.

A análise das vias de impacto se baseia em uma perspectiva de impacto diferente da determinada pelo positivismo na ciência, bus- cando enxergar a complexidade inerente a todo o processo, que envolve feedback e relações entre os sistemas social, cultural e biofísico. Ademais, é válido ressaltar que qualquer método de avaliação de impacto, inclusive a análise das vias de impacto, deve proporcionar um caminho simples para sua compreensão e utilização, facilitando o aprendizado dos envolvidos e permitindo a participação dos atores em geral (BRIONES et al., 2004; SPRINGER-HEINZE et al., 2003).

Compreende-se, então, que o caminho desde o início da pesquisa até seus resultados e a transformação destes em impacto é cumulativo e proveniente da interação entre diferentes elementos e atores. A estruturação de uma análise das vias de impacto se baseia nessa premissa e organiza um mapa que permite visualizar as entradas (inputs), a produção imediata (outputs), os resultados (outcomes), que consideram diferentes graus de abrangência, e os impactos (impacts) (WALKER et al., 2008).

Sendo assim, é elaborada uma representação não linear das vias de impacto, na qual o processo de geração do conhecimento é compartilhado entre os diferentes atores, demonstrando que não é possível fazer uma separação clara entre as partes de cada um deles. Essas análises são conduzidas por meio de estudos de caso, na maioria das abordagens, mas sem perder a capacidade de comparação entre os diferentes casos (JOLY et al., 2015).

A possibilidade de comunicação por meio de um guia visual é um aspecto interessante da análise das vias de impacto. Facilmente, percebem-se os principais resultados e impactos da pesquisa, bem como os atores envolvidos, algo que pode ser bastante positivo para o alcance dos usuários primários, bem como da sociedade em geral (COLINET et al., 2014, 2017).

Existe um robusto corpo de bibliografia contendo estudos de caso que avaliam o impacto das pesquisas com base no modelo das vias de impacto, alguns, inclusive, citados neste ensaio. Embora boa parte deles esteja concentrada na área da agricultura, proporcionando uma 
visibilidade tangível de muitos dos resultados, já existem outras áreas de investigação utilizando essa perspectiva. Um questionamento importante se refere à utilização desse modelo em outras áreas do conhecimento, observando de que forma é possível ter nítida percepção de impactos mais abstratos, abordagem que está a ser explorada em um estudo em andamento.

No momento, não há nenhuma metodologia de avaliação de impacto societal que seja amplamente utilizada ou identificada como um padrão internacional. E, embora a avaliação de impacto da pesquisa não seja uma temática recente, percebe-se que houve um maior interesse pelos aspectos não acadêmicos da contribuição dos projetos e programas científicos na última década. Essas abordagens alternativas têm sido testadas em áreas do conhecimento e esferas institucionais diversas (JOLY et al., 2015).

Todavia, é válido considerar que o discurso dos atores acadêmicos sobre os impactos da pesquisa dificilmente serão considerados completamente imparciais, afirma Hill (2016). Existe todo um contexto que influencia a visão dentro da academia, mas, de toda forma, isso não invalida a necessária discussão sobre os impactos da investigação para a sociedade.

\section{CONSIDERAÇÕES FINAIS}

O propósito deste ensaio teórico foi apresentar um olhar alternativo sobre a avaliação da investigação científica, procurando apresentar a abordagem do impacto societal, sendo capturado por um tipo de análise profunda e complexa conduzida por meio das vias de impacto da pesquisa. É claro que, em apenas algumas páginas, não é possível abordar de forma robusta essa temática. Contudo, espera-se que a apresentação de uma alternativa seja uma contribuição deste trabalho para o debate sobre a avaliação da pesquisa, em especial em um momento de desvalorização da academia e da ciência.

Os atores envolvidos na produção e disseminação do conhecimento, em especial, as agências públicas de financiamento, têm o poder de influenciar a cultura de avaliação de forma a moldar a percepção e o comportamento da comunidade científica. Existem ações institucionais que precisam ser definidas para transformar a orientação a publicações e prestação de contas em uma orientação para o impacto.

Defende-se aqui a perspectiva do impacto societal, que permite a análise de diferentes dimensões e graus de impacto, bem como a compreensão do relacionamento entre eventos, atores e valores no cenário da investigação. Mesmo não havendo um modelo estabelecido e reconhecido como o melhor entre aqueles que debatem esse tema, existe o acordo sobre a necessidade dessa mudança no olhar sobre o processo de avaliação da pesquisa.

A análise das vias de impacto é uma alternativa relevante para esse processo avaliativo, o qual apresenta elementos positivos para toda a esfera científica. A produção de um material robusto e complexo permite aprendizagem e estabelecimento de melhores práticas entre os pesquisadores e gestores científicos, enquanto, também, facilita na comunicação dos resultados e impactos aos atores de fomento e financiamento, bem como para toda a sociedade.

Assim, é possível enxergar muitas possibilidades de estudos dentro dessa discussão. Entre as recomendações, aponta-se que a temática necessita de modelos ideais, de compreensão sobre a comensurabilidade de elementos entre diferentes áreas do conhecimento, além da exploração de uma variedade de métodos e ferramentas que possam retratar de forma confiável o que a teoria sobre impacto societal tem compartilhado.

\section{REFERÊNCIAS}

BALL, S. J. Living the Neo-liberal University. European Journal of Education, v. 50, n. 3, p. 258-261, 2015. Disponível em: http://doi. wiley.com/10.1111/ejed.12132. Acesso em: 15 ago. 2020.

BARRATT, D.; BARRATT, D.; BRENTON, P. Assessment of the Atlas of Living Australia's Impact and Value. Alluvium: [s.n.], 2016. Disponível em: https://www.csiro.au/-/ 
media/About/Files/Impact-assessment/2016/ 2016-ALA-Impact-Evaluation----Alluvium. pdf. Acesso em: 15 ago. 2020.

BORNMANN, L. What is societal impact of research and how can it be assessed? a literature survey. Journal of the American Society for Information Science and Technology, v. 64, n. 2, p. 217-233, 2013. Disponível em: http:// doi.wiley.com/10.1002/asi.22803. Acesso em: 3 mar. 2020.

BOZEMAN, B.; SAREWITZ, D. Public Value Mapping and Science Policy Evaluation. Minerva, v. 49, n. 1, p. 1-23, 2011. Disponível em: https://link.springer.com/content/ pdf/10.1007/s11024-011-9161-7.pdf. Acesso em: 3 mar. 2020.

BRIONES, R. et al. Impact pathway analysis for research planning: The case of aquatic resources research in the WorldFish Center. NAGA, WorldFish Center Quarterly, v. 27, n. 3/4, p. 51-55, 2004. Disponível em: http:// www.worldfishcenter.org/content/impact-pathway-analysis-research-planning-case-aquatic-resources-research-worldfish-center-0. Acesso em: 3 mar. 2020.

COLINET, L. et al. ASIRPA - Analyse des Impacts de la Recherche Publique Agronomique. Rapport final. Paris, France: [s.n.], 2014. Disponível em: https://hal.archives-ouvertes.fr/hal-01064096/. Acesso em: 3 mar. 2020 .

COLINET, L. et al. Des barèmes génériques pour évaluer les impacts de la recherche sur la société: l'exemple des impacts politiques. Cahiers Agricultures, v. 26, n. 6, 2017. Disponível em: https://hal.archives-ouvertes.fr/hal01676197/. Acesso em: 3 mar. 2020.

DOUTHWAITE, B. et al. Impact pathway evaluation: An approach for achieving and attributing impact in complex systems. Agricultural Systems, v. 78, n. 2, p. 243-265, 2003. Disponível em: https://doi.org/10.1016/S0308521X(03)00128-8. Acesso em: 19 abr. 2020.
GRANT, J. et al. Capturing Research Impacts. RAND Europe. 2010. Disponível em: https://www.rand.org/pubs/documented_briefings/DB578.html. Acesso em: 5 abr. 2020.

GUNN, A.; MINTROM, M. Evaluating the non-academic impact of academic research: design considerations. Journal of Higher Education Policy and Management, v. 39, n. 1, p. 2030, 2017. Disponível em: https://doi.org/10.1080/1 360080X.2016.1254429. Acesso em: 14 fev. 2020.

HILL, S. Assessing (For) impact: Future assessment of the societal impact of research. Palgrave Communications, v. 2, p. 1-7, 2016. Disponível em: http://dx.doi.org/10.1057/palcomms.2016.73. Acesso em: 5 ago. 2020.

JOLY, P. B. et al. ASIRPA: A comprehensive theory-based approach to assessing the societal impacts of a research organization. Research Evaluation, v. 24, n. 4, p. 440-453, 2015. Disponível em: https://doi.org/10.1093/reseval/ rvv015. Acesso em: 5 ago. 2020.

JOLY, P.B.; MATT, M. Towards a new generation of research impact assessment approaches. Journal of Technology Transfer, p. 1-11, 2017. Disponível em: https://link.springer.com/ article/10.1007/s10961-017-9601-0. Acesso em: 5 ago. 2020.

KUBY, T. Innovation is a social process. What does this mean for impact assessment in agricultural research? Eschborn, Germany: CIAT workshop, 1999.

MATT, M. et al. Opening the black box of impact - Ideal-type impact pathways in a public agricultural research organization. Research Policy, v. 46, n. 1, p. 207-218, 2017. Disponível em: http://dx.doi.org/10.1016/j.respol.2016.09.016. Acesso em: 5 ago. 2020.

MOLAS-GALLART, J. Research Governance and the Role of Evaluation. American Journal of Evaluation, v. 33, n. 4, p. 583-598, 2012. Disponível em: http://journals.sagepub.com/doi/10.1177/1098214012450938. Acesso em: 5 ago. 2020. 
MOLAS-GALLART, J. Research evaluation and the assessment of public value. Arts and Humanities in Higher Education, v. 14, n. 1, p. 111-126, 2015. Disponível em: https://doi. org/10.1177\%2F1474022214534381. Acesso em: 5 ago. 2020.

SPRINGER-HEINZE, A. et al. Impact pathway analysis: an approach to strengthening the impact orientation of agricultural research. Agricultural Systems, v. 78, n. 2, p. 267-285, 2003. Disponível em: https://doi.org/10.1016/ S0308-521X(03)00129-X. Acesso em: 5 ago. 2020.

TERÄMÄ, E. et al. Beyond academia - Interrogating research impact in the research excellence framework. PLOS ONE, v. 11, n. 12, p. 1-19, 2016. Disponível em: https://doi. org/10.1371/journal.pone.0168533. Acesso em: 5 ago. 2020.

TERRAPON-PFAFF, J. et al. Impact pathways of small-scale energy projects in the global south - Findings from a systematic evaluation. Renewable and Sustainable Energy Reviews, v. 95, p. 84-94, 2018. Disponível em: https://doi.org/10.1016/j.rser.2018.06.045. Acesso em: 5 ago. 2020.

THOMAS, D. A. et al. Changing research on research evaluation: a critical literature review to revisit the agenda. Research Evaluation,v. 29, n. 3, p. 275-288, 2020. Disponível em: https://doi.org/10.1093/reseval/rvaa008. Acesso em: 5 ago. 2020.

WALKER, T. et al. Strategic Guidance for Ex Post Impact Assessment of Agricultural Research. Rome, Italy: [s.n.], 2008. Disponível em: https://ispc.cgiar.org/sites/default/files/ ISPC_SPIA_StrategicGuidance.pdf. Acesso em: 5 ago. 2020. 